\title{
Time discrimination training of laboratory animals using a PDP-8 computer
}

\author{
BARRY L. JOHNSON and W. KENT ANGER \\ Behavioral Studies Laboratory \\ Behavioral and Motivational Factors Branch, DLCD \\ National Institute for Occupational Safety and Health \\ 1014 Broadway, Cincinnati, Ohio 45202
}

The purpose of this paper is to describe the use of a small, general-purpose digital computer system in the training of laboratory animals to discriminate stimuli on the basis of their duration. The computer hardware and software required for such training, together with some illustrative results from two recent studies, will be described.

Our interest in time discrimination evolves from an effort to structure a behavioral toxicology program useful for laboratory testing of the toxicity of contaminants found in community and industrial atmospheres. Such a program, we feel, should range from use of the standard schedules of reinforcement to use of performance tasks involving complex decisions based on the informational content of the stimulus. In addition, the elements of the behavioral testing program should include behaviors that are representative of necessary human behavior. For these reasons, we have included time discrimination performance in our behavioral toxicology testing profile.

The utilization of laboratory animals in behavioral toxicology testing is required for the following types of studies: (1) those requiring maximum environmental control. (2) exposure levels in excess of limits thought safe for humans, and (3) exposure to agents whose toxicity is unknown. Three female monkeys (Macaca mulatta) have served as $\mathrm{Ss}$ for the time discrimination performance reported here.

The utilization of a digital computer in our time discrimination performance was dictated by the lack of published reports concerning this type of behavior in laboratory animals. It was. therefore, felt desirable to structure the behavioral performance around equipment

* The authors are pleased to acknowledge the participation of Dr. Charles Xintaras and James $V$. Setzer in the conduct of the work reported in this paper. that would provide the $\mathrm{E}$ with maximum flexibility in behavioral control. The power of the on-line digital computer in controlling behavioral experiments is now well recognized and needs no further elaboration by us.

\section{EQUIPMENT}

\section{Behavioral Equipment}

The behavioral testing chamber was a modified laboratory animal exposure chamber commonly used in toxicity studies. The chamber's interior dimensions were approximately $92 \times 84 \times 84 \mathrm{~cm}$. One wall of the chamber contained a test panel with the stimulus-response manipulanda. The general arrangement of this panel is shown in Fig. 1. A 4-in.-sq translucent plastic window which could be backlighted for the presentation of visual stimuli was situated in the center of the test panel. Behind this window were placed 25 miniature incandescent lamps (General Electric No. 1819) arranged in a 5 by 5 matrix. Each lamp could be individually lighted upon computer command. The visual stimulus routinely consisted of a $2 \times 2$ square pattern.
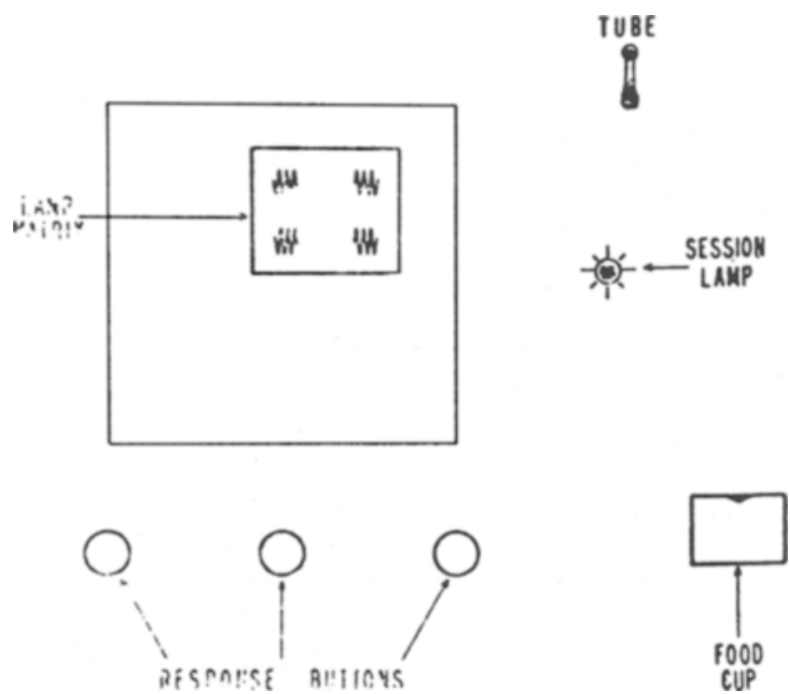

Fig. 1. Schematic arrangement of the behavioral testing panel. 
Situated below the visual stimulus window were three button response switches (Gerbrands Model E8670A) used by the $S$ to initiate and respond to stimuli. These switches could also be backlighted. a feature which was used to signal the $S$ as to the order of responses. For example, a backlighted center switch served to cue the animal that a response on the center switch would be followed by presentation of a stimulus. All three response switches were backlighted during and following the presentation of a stimulus for discrimination and remained lighted until the $S$ made a choice response or until a limited hold period was exceeded.

Auditory stimuli were presented to the $S$ by gating the output from a $430 \cdot \mathrm{Hz}$ fixed-frequency oscillator into a loudspeaker fixed to the back of the testing panel. The electronic gate consisted of a transistor switch driven by an enabling pulse from the computer's AX08 laboratory peripheral unit. A similar arrangement, using the same loudspeaker, provided two distinct types of auditory cues used to signal error conditions. Both types of cues utilized a gated fixed-frequency tone of $1,600 \mathrm{~Hz}$. Whenever the $S$ made an incorrect choice response (i.e., an incorrect discrimination), the tone was presented as a continuous stimulus for a length of time specified by the $\mathrm{E}$ at the beginning of the session. The second type of error-produced cue was given whenever the $S$ made some type of procedural error such as making a response while the time stimulus was being presented. For this case, the $1,600 \cdot \mathrm{Hz}$ tone was gated on and off every $125 \mathrm{msec}$, thus producing a modulated effect readily distinguishahle from the continuous stimulus $(1,600-\mathrm{Hz}$ tone) associated with an incorrect choice response.

Also attached to the testing panel (Fig. 1) were a holding bar, a food pellet dispenser, and a session lamp. The holding bar was used to position the $S$ with respect to the panel and to prevent the $S$ from making responses with either hand. A food dispenser (Gerbrands Model D-1) dispensed banana-flavored pellets (Noyes, $190 \mathrm{mg}$ ) for reinforcement. The session lamp, when enabled, informed the $S$ that an experimental session was underway.

The S's behavior during each experimental session was monitored using a closed-circuit television camera positioned adjacent to the testing chamber.

\section{Computer Hardware}

The computer system utilized in the work reported here was a PDP-8/I computer arranged in a LAB-8 configuration. The system included a central processor unit containing 8,192 core memory registers, a 60-character/sec (cps) paper-tape punch, a 300-cps paper-tape reader, a 10-cps teleprinter/typewriter. a Tektronix Model 504 oscilloscope, two channels of digital-to-analog conversion. and a programmable real-time clock (KW8/IE) calibrated in 1 -msec intervals. A lamp controller interface supplied by the Digital Equipment Corporation permitted individual control over each of the 25 lamps situated in the testing panel.
The relay outputs (R1.R2. R4) of the LAB-8's AX08 peripheral unit were used to control the status of the food pellet dispenser, tone generators. and session cue lamp. All switch closure responses made by the $\mathrm{S}$ were filtered by analog switch closure filters whose outputs were sensed by the computer via the program interrupt facility. A system such as the one just described offers the $\mathrm{E}$ capabilities for behavioral equipment control, data display, data recording and manipulation, timing operations, and communication with the behavioral session.

\section{BEHAVIORAL TRAINING AND COMPUTER SOFTWARE}

The Ss were trained using a series of seven computer programs that shaped the animals periormance in steps of increasing complexity ranging from food pellet dispensing to the final time discrimination behavior. Because the computer programs were written to achieve specific steps in the animals' training, the training sequence and computer software are discussed jointly. Prior to discussing the individual training programs, it should be mentioned that two points bear emphasis regarding all software development. First, it was recognized at the outset that no one computer program could be written that would incorporate all the behavioral training contingencies necessary to shape an animal's performance from the naive state to the final time discrimination performance. The decision was therefore made to shape the S's behavior in a series of steps, with each step requiring a different computer program. In order to reduce the programming burden as much as possible, each program was written as a series of interlocking subroutines. Using this approach permitted the incorporation of common subroutines into several training programs. This approach avoided the necessity of having to rewrite large segments for each new program.

The second important consideration inherent in our software development was to include as many options as possible for the E's use in changing certain parameters of the experimental session. This permitted the $E$ to alter given training steps in the manner most appropriate for each animal.

A brief description of each training program follows. Use of these programs will permit training of a naive animal to the point of time uiscrimination performance for both visual and auditory stimuli in approximately 4 months. This assumes two training sessions of $45 \mathrm{~min}$ duration per day per animal.

\section{Program 1 (Magazine Training)}

The animal was trained to obtain. at the sound of the feeder, food pellets delivered every $60 \mathrm{sec}$. The computer program required the $E$. at the start of each session, to specify the total number of food pellets to be delivered. The computer displayed on the oscilloscope a running record of the number of pellets delivered. This 
program, though simple in concept. required writing several general-purpose subroutines (such as teletypewriter/printer input and output routines and an oscilloscope display routine) which were used in all subsequent programs.

\section{Program 2 (Buttonpress and Bar Holding)}

This program was used by the $\mathrm{E}$ to shape buttonpress responses and to train the $S$ to hold down the holding bar. The program provided the $E$ with an option to choose between reinforcing all button responses or only those made on backlighted response switches. The $E$ could control food reinforcement by a remote switch that actuated the food dispenser. A general-purpose subroutine written to identify all switch responses, including a release of the holding bar (which was connected to one of the 15 digital inputs of the behavioral interface), was written for this program and used in all subsequent programs.

\section{Program 3 (Stimulus Duration Selection)}

Under control of the $\mathrm{E}$, visual stimuli having durations of either 0.5 or $3.5 \mathrm{sec}$ were presented to the $\mathrm{S}$. The correct response switch for the 0.5 -sec duration stimulus was the left button, and the right switch was correct for the $3.5-\mathrm{sec}$ stimulus. Correct responses were reinforced with food pellets and incorrect responses were ignored. The $\mathrm{E}$ presented the desired stimulus by commands to the program entered via the teletypewriter. This program incorporated a subroutine written to process the programmable real-time clock which was used to time the duration of all stimuli.

\section{Program 4 (Self-Pacing Behavior)}

At this step, the $S$ was trained to initiate the stimulus presentation, i.e., to self-pace the time discrimination performance. This was achieved by having the animal press the center of the three response buttons in order to request a computer presentation of a $0.5-$ or $3.5 \mathrm{sec}$ stimulus. In addition, a prestimulus delay of $250 \mathrm{msec}$ preceded the stimulus occurrence. This delay was included in order to give the animal time to look toward the visual stimulus panel. Also, a short poststimulus delay of $100 \mathrm{msec}$ followed the stimulus presentation in order to preclude any reinforced responses when the stimulus was still present. Incorrect responses were ignored at this step in training.

\section{Program 5 (Time Out from Reinforcement)}

This step introduced time out (TO) from reinforcement whenever incorrect choice responses were made. Each response made during a TO period reset the TO clock to the maximum value. The $E$ specified the length of the TO period via the teletypewriter at the beginning of each experimental session. An initial value of $5 \mathrm{sec}$ was used. a value which was gradually increased throughout the remaining training programs. This program also permitted providing TOs for responses made during the stimulus presentation period and the delay periods that preceded and followed each stimulus.

\section{Program 6 (Center Switch Responding)}

At this step in training, a visual stimulus of $2 \mathrm{sec}$ duration was introduced for about $20 \%$ of the total stimulus presentations. The remaining stimuli were either 0.5 or $3.5 \mathrm{sec}$ in duration. The animals were trained to respond on the center switch whenever a $2-\mathrm{sec}$ stimulus appeared. A correct response for this situation was not food reinforced, but rather was used to advance the $S$ to the next stimulus to be drawn from a library of possibilities stored within the computer's core memory. The program provided an option by which the $E$ could either ignore or punish with a TO all incorrect responses made for stimuli of $2 \mathrm{sec}$ duration.

\section{Program 7 (Time Discrimination Performance)}

This program was used to shape the final step of time discrimination behavior. This step involved training all Ss to discriminate both auditory and visual stimuli on the basis of stimulus duration. Prior to this point in training, only visual stimuli had been presented for discrimination. By pairing an auditory stimulus of $430 \mathrm{~Hz}$ frequency with the visual stimulus, and then by gradually decreasing the visual stimulus intensity, it was possible to shape responding to auditory stimuli. This step was achieved by placing in front of the visual stimulus an adjustable polarized optical filter that could be manually positioned to diminish the stimulus intensity.

In addition, two other elements were added to the Ss' performance in this stage of training. First, a limited hold was imposed on all choice responses. This contingency required the animal to respond within a specified length of time after stimulus termination or be punished with a time-out. The limited hold was added to the animals' training in order to minimize response latency variability. This particular TO was in response to a procedural error, so the modulated tone was presented. Also. the percentage of $2-\mathrm{sec}$ stimuli was gradually increased from $20 \%$ to $70 \%$.

\section{Final Performance}

The final time discrimination performance resulting from Program 7 is summarized in Fig. 2, which illustrates the sequence of events in the performance. As mentioned previously, the task is self-paced and commences when the animal presses the backlighted center button. A brief prestimulus delay of $250 \mathrm{msec}$ follows. during which all response switches are backlighted. An auditory or visual stimulus of variable duration is then presented. The stimulus duration which was presented could assume one of three possible values: $2 \mathrm{sec}$, less than $2 \mathrm{sec}$. or greater than $2 \mathrm{sec}$. The durations of the stimuli less than or greater than $2 \mathrm{sec}$ were established by the $\mathrm{E}$ at the beginning of each experimental session. The order of occurrence of stimuli 


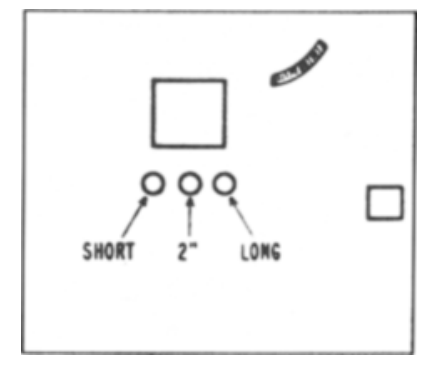

Fig. 2. Sequence of events in the time discrimination performance.

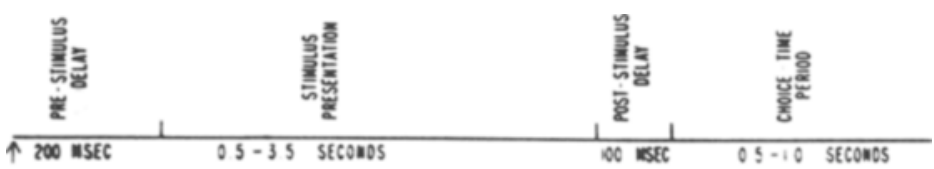

\section{Cus - Pacoucia:}

itseose (CN)

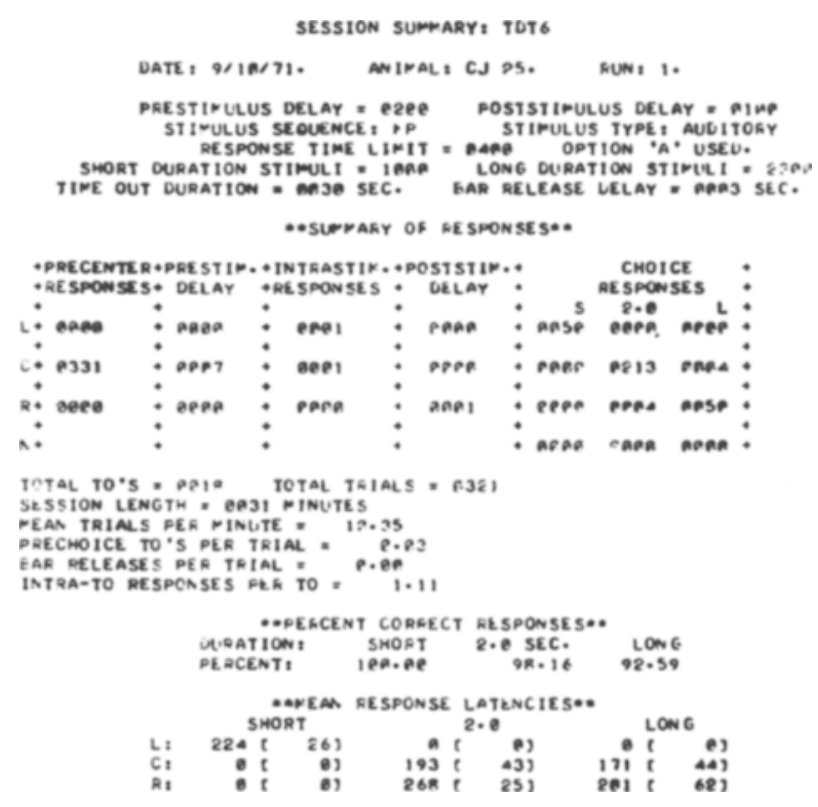

Fig. 3. Typical printout of summary results obtained for each testing session.

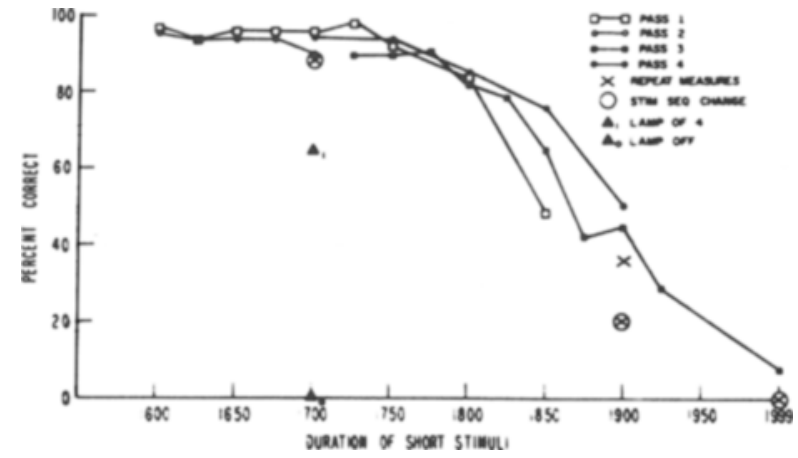

Fig. 4. Effect of increased difficulty of discrimination on percent correct responses. was semirandomized and drawn from various stimulus libraries of 300 elements stored in the computer's memory. The starting position within the stimulus library was also varied for each experimental session. A brief poststimulus delay of $100 \mathrm{msec}$ followed the stimulus presentation. After this delay, the $S$ was required to respond within the limited hold period. All correct responses for short or long duration stimuli were reinforced with food pellets; correct responses following a 2-sec stimulus were not reinforced with food. All responses made during the prestimulus or poststimulus delays, or during stimulus presentations, as well as failure to respond within the limited hold period, were punished with the "beeping" TO lasting 15-30 sec, depending on the animal being tested. An incorrect choice response was punished with a TO of equal duration, but with a continuous tone.

\section{Data Acquisition}

Several measures characterizing the S's performance were recorded for each session. A summary of these data was printed on the teleprinter at the end of each session and consisted of the following: number and percent of incorrect responses, number of responses made during the TO, intrastimulus (or delay) periods, number of stimuli generating no responses within the length of session, total limited hold period, number of choice responses, and mean choice response latencies with their associated standard deviations. These latter data measured the elapsed time from the termination of the stimulus to the time the animal made a choice response. A typical printout from an experimental session is shown in Fig. 3. The data computations indicated on the summary printout shown in Fig. 3 were performed using DEC's floating point package. The response data were used to characterize each $\mathrm{S}$ 's session performance. By changing the degree of difficulty of the discrimination. we obtained data on the limits of our time discrimination performance. A typical result is shown in Fig. 4. Which illustrates the deterioration of correct 
performance (following corrections for guessing) as the discrimination became more difficult.

In addition to the summary printout, a punched paper-tape record was obtained at the end of each session. This record contained all responses made by the $\mathrm{S}$ in the order in which they were made. Associated with each response was the response latency from that trial. The paper tapes were punched in ASCII format for subsequent analysis by FORTRAN programs requiring this type of input format. The FORTRAN package available in DEC's PS-8 operating system was used for data analysis. One type of analysis involves histogram classification of the response latencies. Typical results are shown in Figs. 5 and 6, which illustrate response latency histograms printed on the teleprinter. The histogram analysis program permits the $E$ to select the number of data bins and the bin width and to set maximum and minimum bounds on the latencies of interest. Figure 5 indicates the distribution of response latencies made on the rightmost switch for long duration

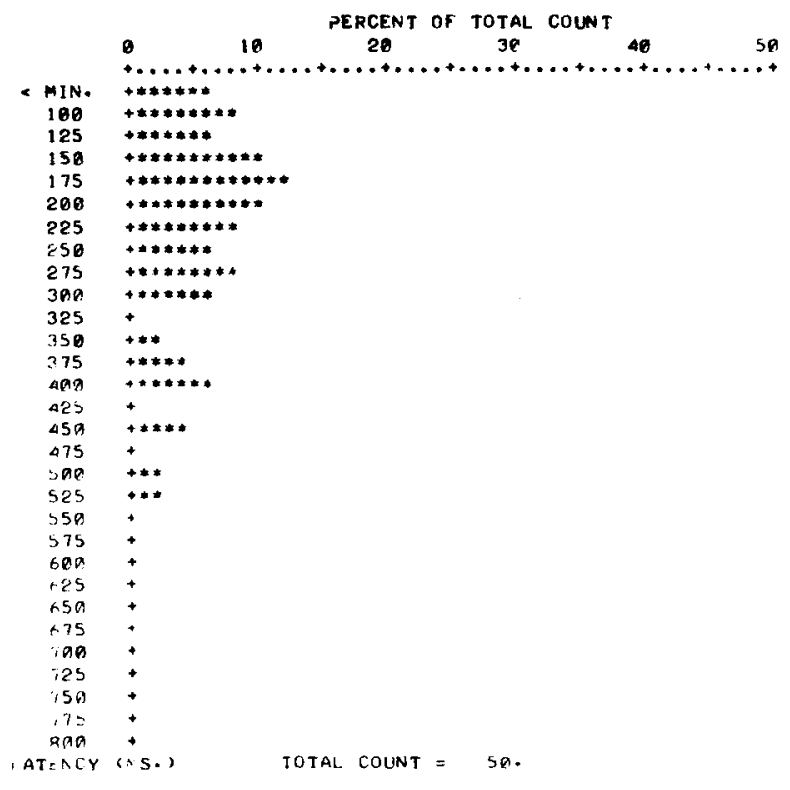

Fig. 5. Histogram of response latencies for $S 25$ for responses made with the rightmost button in response to visual stimuli of 2,200 msec duration.

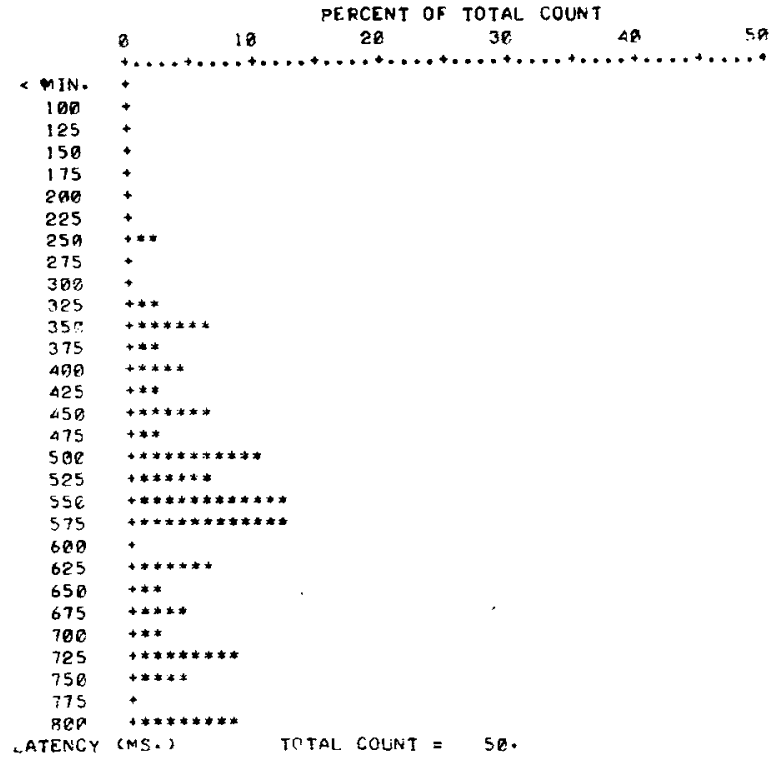

Fig. 6. Effect of $3 \mathrm{mg} / \mathrm{kg}$ of chlorpromazine on response latencies made in response to visual stimuli of $2,220 \mathrm{msec}$ duration for S 25. Behavioral testing was conducted $3 \mathrm{~h}$ following drug administration (oral).

stimuli. Figure 6 illustrates the effect of a $3-\mathrm{mg} / \mathrm{kg}$ dose of chlorpromazine on response latencies from the same $S$ on the following day.

\section{SUMMARY}

A time discrimination performance using rhesus monkeys has been structured as part of a general behavioral toxicology testing program. By using an on-line computer, three Ss were trained in time discrimination performance using seven training steps. Each training step used a separate computer program built upon the program which preceded it in the steps of training. By writing each program as a series of interlocking machine-language subroutines, complex training programs were assembled with minimum effort. Our experience indicates that the use of an on-line computer in psychological investigations provides the $\mathrm{E}$ with considerable flexibility in behavioral control and prompt knowledge of summary results. 